BIO Web of Conferences 1, 00088 (2011)

DOI: $10.1051 /$ bioconf $/ 20110100088$

(C) Owned by the authors, published by EDP Sciences, 2011

\title{
Multisensory interaction in vibrotactile detection and discrimination of amplitude modulation: insights from expert MFS surgeons and naive participants
}

\author{
Kinneret Teodorescu $^{*} \quad$ Sylvain Bouchigny ${ }^{\#} \quad$ Pablo Hoffmann $^{* *}$ \\ *Technion, Haifa, Israel \\ ${ }^{\#}$ CEA, LIST, Paris, France \\ **Aalborg University, Denmark. \\ E-mail:kinneret_w@yahoo.com
}

Maria Korman*

\begin{abstract}
Perception of vibration during drilling demands integration of haptic and auditory information with force information. In this study we explored the ability to detect and discriminate changes in vibrotactile stimuli amplitude based either on purely haptic feedback or together with congruent synthesized auditory cues in groups of naive subjects and expert surgeons. Our results point toward the complex influence of multimodal experience during vibration perception. First, in naive subjects, we showed that detection and discrimination of amplitude change in complex vibro-tactile stimulus is selectively sensitive to combination of modality and previous experience. In the domain of discrimination, our results suggest that bi-modal performance is always better than uni-modal performance regardless of order of experience. Second, experiments with expert surgeons revealed that expertise in complex skill of maxilla-facial surgery strongly relies on enhanced touch perception, as measured in reaction times and discrimination ability in bi-modal vibro-auditory conditions. These observations suggest that acquisition of mandibular surgery skill has brought to an enhanced representation of vibro-tactile modulations in relevant stimuli ranges. Altogether, our results provide basis to assume that during acquisition of mandibular drilling skill, trainees may benefit from training of relevant basic aspects of touch perception - sensitivity to vibration and accompanying modulations of sound.
\end{abstract}

\section{Introduction}

Auditory-tactile perception of vibratory stimulation is a unique type of multisensory interaction in which the two sensory modalities involved are sensitive to the same kind of physical property - mechanical pressure in the form of oscillations, were the very same vibratory stimulus can be experienced simultaneously by the peripheral receptor organs of both sensory modalities (the basilar membrane in the cochlea and the skin) (Soto-Faraco\&Deco, 2009). Sensory quality of multisensory events is often dominated by one of the two modalities (e.g., visual dominance principle), however, recent studies suggest that the degree of dominance is determined by the statistical reliability of the available sensory information (Ernst\&Banks, 2001). In the audio-vibrotactile domain there are demonstrations of tactile influences on the perception of sound (Bresciani et al., 2007, Caclin et al., 2002, Murray et al., 2005), and in contrary, of acoustic influence in the perception of touch (Soto-Faraco et al., 2004, Bresciani et al., 2005). Multisensory integration of auditory and visual with tactile information is most efficient when the different sensory stimuli are relevant, temporally and spatially coincident or 'contiguous'. Recent evidence, however, suggest that that spatial contiguity may be less relevant for tactileauditory integration as compared to visuo-tactile (Zampini et al., 2007, Gillmeister et al., 2007).

Surgery often involves tool-tissue interactions producing vibrations and sounds that may serve as a primary source of sensory information for correct surgical performance, especially in the cases when visual information is unavailable due to occlusions in the working field (Praamsma et al., 2008). In such cases the surgeon must rely on vibro-tactile and auditory sensory modulations to detect and discriminate critical steps in the surgical procedure. Modulation of intensity is a basic perceptual dimension where stimuli can be compared along the intensive continuum as long as they impinge upon the same sensory source. In a previous series of studies on another aspect of touch, stiffness discrimination (Korman et al., 2011), we have reported that for suprathreshold comparison pairs multimodal cue significantly affected stiffness discrimination ability, depending on the matching between the modalities and

This is an Open Access article distributed under the terms of the Creative Commons Attribution-Noncommercial License 3.0, which permits unrestricted use, distribution, and reproduction in any noncommercial medium, provided the original work is properly cited. 
the order of experience. Since the global aim of this research is to find the ways of perceptual enhancement of surgeons in concrete stages of jaw surgery, in the current study we addressed the question of how the perceiver, either naïve or expert, integrates information from tactile, auditory or both matched modalities to detect and discriminate between vibrotactile stimulus amplitudes.

\section{Method}

Participants and setup. A total 60 naive right handed subjects, generally healthy, with normal or corrected to normal vision, served as paid participants in the experiment (medicine student and other professionals specifically trained to palpate objects were excluded). In addition, six expert surgeons from different hospital in France took part in the study.

The experimental setup included a computer screen, a vibrating handle and three answering buttons. The subjects were asked to sit in front of the computer holding the vibrating handle with their right hand and with three fingers as in handwriting or drawing. Their right arm rested on an armchair support. Their left hand was used for answering using three buttons of the answering device. A noise reduction headset (with a noise reduction of $-31 \mathrm{db}$ ) was used to play the white noise to the subject during vibro-tactile stimuli presentation of No Sound condition to isolate completely the vibrating handle sound.

The vibrating handle was specifically designed for the MFS demonstrator with an overall form factor as close as possible to a real drilling tool used in bone surgery. The stimulus was provided by 12 longitudinal beams manufactured at the surface of the handle and actuated by a stack piezoelectric transducer. The beams produced a radial vibration in every direction around the stylus (with a cylindrical symmetry) and their specifications were optimized to allow stimulating the skin's mechanoreceptors within a wide range of frequencies ( $1 \mathrm{kHz}$ bandwidth) and amplitudes (up to $100 \mu \mathrm{m})$.

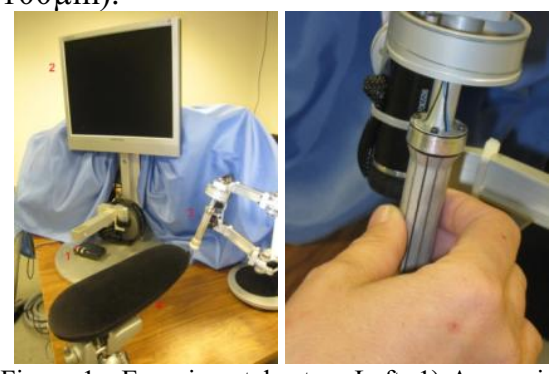

Figure 1 : Experimental setup. Left: 1) Answering button device, 2) computer screen, 3) Vibrating handle, 4) Arm support. On the right, the picture shows how the subject is told to hold the handle.

The vibrating stimuli were chosen according to real captured data from the operating room. The main excitation perceived by the surgeon's hand is the fundamental vibration generated by the drill rotation and its first harmonic. For a drill rotating at $12000 \mathrm{rpm}$ the two frequencies are $200 \mathrm{~Hz}$ and $400 \mathrm{~Hz}$. These two frequencies have the highest amplitudes, while the other frequencies have much lower amplitudes. Captured data analysis revealed that significant changes in relative amplitude at several specific frequencies occur during punctual drilling. In the beginning, surgeons experience vibrating stimuli mainly consisting of two frequencies $(400 \mathrm{~Hz}$ and $200 \mathrm{~Hz}$ ) with high amplitudes (about $10 \mu \mathrm{m}$ ), and during the drilling and especially and the end of the drilling, the amplitude of the $400 \mathrm{~Hz}$ frequency depends greatly on the surgeon behavior and the bone density or geometry, while the amplitude of the $200 \mathrm{~Hz}$ frequency keeps its high amplitude. The baseline amplitude of the stimulus was $10 \mu \mathrm{m}$. The tactile stimuli were defined as follows: The signal is the additive synthesis of two sinusoidal waveforms. One at $200 \mathrm{~Hz}$ with constant normalized amplitude of $1(10 \mu \mathrm{m})$ and a $400 \mathrm{~Hz}$ waveform with varying relative amplitude from 0.3 up to 0.95 . These two signals simulated the vibrations generated in the tactile domain by a drill rotating at $12000 \mathrm{rpm}(200 \mathrm{~Hz})$ and corresponded respectively to the fundamental and the first harmonic of the excitation. The sound signal played to the subject in the headset was a flat distribution of white noise filtered at the frequency of $200 \mathrm{~Hz}$ added to a flat distribution of white noise filtered at $400 \mathrm{~Hz}$. Relative amplitudes of the two signals varied in the same way as the tactile excitation.

Task, design, and procedure. Subjects were instructed to hold the vibrating handle and press a response button as fast as possible after the change in vibration intensity is detected by the participant. Immediately after the trial ended (by pressing the response button or automatically after $4 \mathrm{sec}$ from the beginning of the trial), the following question was presented on the screen: "Which of the stimuli was more intense, the first or the second one?" Stimulus with higher $400 \mathrm{~Hz}$ frequency amplitude was expected to be perceived as more intense. To report his choice the participant pressed one of three buttons, corresponding to: "the first stimulus", "the second stimulus" or "I don't know" answers. After pressing on the relevant answer key, a black screen was shown, with the sentence "be ready" for 1 second.

One of the two stimuli (V1 or V2) was always kept constant, and served as at the baseline comparison value. This value was chosen to consist of 2 frequencies, $400 \mathrm{~Hz}$ and $200 \mathrm{~Hz}$ with amplitude around $10 \mu \mathrm{m}$ each. The second stimulus was one of 11 possible comparison values chosen in 5\%-10\% intervals from the amplitude of the $400 \mathrm{~Hz}$ frequency in 
the baseline. The comparison values were chosen to create a range of discrimination scores, starting from roughly chance level and up to near perfect discrimination. The varying relative amplitude ranged from 0.3 up to 0.95 per cents from baseline stimulus

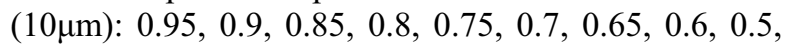
0.4 and 0.3 . Baseline and comparison values were pseudo-randomized, such as for half of the trials the baseline was the first stimulus and the comparison value was the second, and vice versa. Each comparison pair randomly repeated 16 times during the block. The total number of trials in each block was $16^{*} 11=176$. If the response was provided before the second stimulus was presented, this trial was marked as an "error trial" and was repeated at the end of the same test block. The total duration of each trial was max 4 seconds. The trial began with the presentation of the first vibration level, and ended when the participant pressed the response button. To avoid anticipation in the timing of the second stimulus, duration of the first vibration, T1, was shuffled. T1 length was uniformly distributed between 0.8 to 1.4 seconds. The maximal duration of the second stimulus was 4 seconds minus T1.

All subjects performed the task in two consecutive blocks, separated by $5 \mathrm{~min}$ rest. The order of block presentation was counterbalanced between groups. In each trial participants experienced vibration produced by the handle that was composed of two concatenated levels of vibration amplitude.

Testing conditions differed with regard to the sensory information available. In the vibro-tactile $\mathbf{V}$ condition (non-matched auditory information) the only information available for subjects regarding the change in vibration was in the haptic modality. In the vibrotactile- auditory VA condition (matched vibro-tactile and auditory information), in addition to the vibrotactile changes, corresponding auditory information was available, provided by the means of the rendered sound from the speakers/earphones. The auditory information was congruent with the vibro-tactile information, so that rendered auditory stimuli consisted of the same frequencies and were of the same amplitudes as in the vibrating stimulus. In the auditory A condition (non-matched vibro-tactile information) only the auditory component was changing according to the comparison values, while vibro-tactile component was kept constant at the baseline level. Each participant experienced two separated detection blocks. The order of blocks was counterbalanced across participants.

Experiment I - control (12 persons): we tested the baseline effects of practice in VA1-VA2 group. Same vibro-auditory conditions were presented in the two successive performance blocks.
Experiment II - (12 per group). To address the question how the order of experiencing uni- or bi-modal stimuli affects vibration detection and discrimination, four groups of subjects were tested as follows: two groups of subjects were tested in matched vibro-auditory cue vs. vibro-white noise cue: VA1-V2 (vibro-auditory first - vibro second), V1-VA2 (vibro first - vibroauditory second); and two groups were tested on vibroauditory cue vs. auditory only cue: VA1-A2 (vibroauditory first - auditory second) and A1-VA2 (auditory first - vibro-auditory second).

Experiment III: Six expert MFS surgeons were tested in V1-VA2 condition. One surgeon was tested in additional VA block with two pairs of surgical gloves (like during real surgery) to address the question how limitation in vibrotactile input affects performance of an expert.

Performance measures were calculated as follows: detection - percentage of correctly detected changes in stimuli amplitude from all trials in a given block; RT reaction time, measured per trial as time of pressing the response button after second stimulus appearance, in msec; discrimination - percentage of correctly discriminated pairs of stimuli relative to all trials in a given block, indicative of the direction of the change. The data of experiment I and II were analyzed in a within-subjects design using repeated measures ANOVA with groups and blocks as variables. The results of experiment III were analyzed using a MannWhitney $U$ test, to account for different sample sizes.

\section{Results}

In this paper only the RT and PC results are presented Naive participants

RT: Experiment I: No significant difference in RTs of the two performance blocks in VA1-VA2 control group at all difficulty levels were found, indicating that no practice-induced improvement happened by the second block in bi-modal condition in terms of reaction time. Experiment II: Uni-modal to bi-modal transitions were accompanied by overall significant enhancement in performance for A1-VA2 group $(F(1)=15.02$, $\mathrm{p}=0.003$ ) and no difference between blocks for V1VA2 group. In contrast, in bi-modal to uni-modal transitions, for both groups, VA1-A2 and VA1-V2, no overall difference in performance of the two blocks was found.

Discrimination: Experiment I: The results showed no significant difference in percentage of correctly discriminated pairs at all difficulty levels between the two performance blocks in VA1-VA2 control group. In line with observations in the domain of detection for the same group, results suggest that practice in the amount provided by itself does not result in 
improvement in the second block for bi-modal condition. Experiment II: In both groups where bimodal block followed the uni-modal practice, performance in bi-modal condition was significantly better $(\mathrm{GLM}, \mathrm{F}(1)=14.76, \mathrm{p}=0.003$ and $\mathrm{F}(1)=10.63$, $\mathrm{p}=0.008, \mathrm{~A} 1-\mathrm{VA} 2$ and $\mathrm{V} 1-\mathrm{VA} 2$, respectively). In the VA1-A2 and VA1-V2 groups, where uni-modal condition, either auditory or vibro-tactile, followed the bi-modal block, again, significant difference between conditions was found (GLM, F(1)=12.02, $\mathrm{p}=0.005$ and $\mathrm{F}(1)=8.54, \quad \mathrm{p}=0.014, \quad \mathrm{VA} 1-\mathrm{A} 2$ and VA1-V2, respectively) in favor of bi-modal condition. Thus, in terms of percentage of correctly discriminated pairs, bi-modal performance was significantly better regardless of order of experience.

\section{Surgeons}

Experiment III: We have tested six expert MFS surgeons in V1-VA2 condition. The results point toward significant influence of expertise in complex sensory-motor task on basic vibro-tactile perception. Overall, experts exhibited significantly faster bi-modal RT of detection, as compared to naive subjects from the V1-VA2 group in experiment II (Fig.2). In terms of $\mathrm{PC}$, a trend for better discrimination ability was observed, but didn't reach significant differences, given the low number of participants in the surgeons group and the high variance across participants.

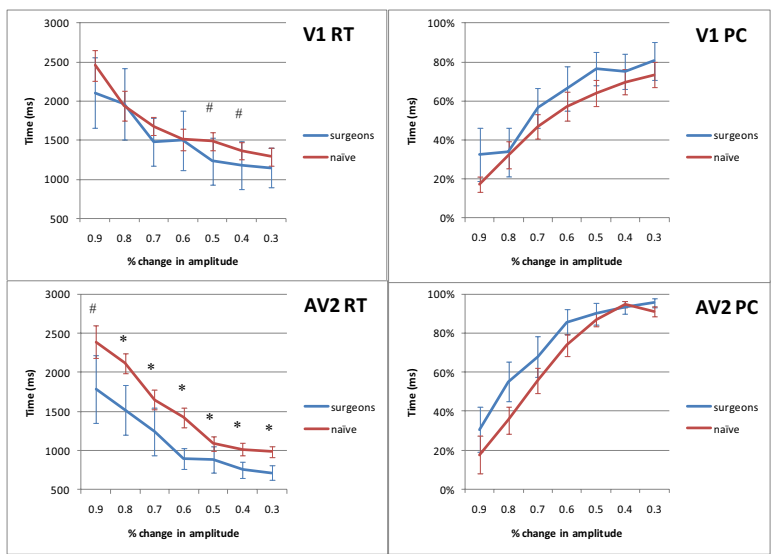

Figure 2. Mean RT (left) and PC (right) for surgeons (blue) and naive participants (red) \pm SE. Significant differences between surgeons and naive participants are denoted by * for $\mathrm{p}<0.05$ and by \# for $\mathrm{p}<0.1$.

\section{Conclusions}

The current study has focused on cross-modal effects and effects of order of experience on vibro-tactile amplitude detection and discrimination. We showed that detection and discrimination of amplitude change in complex vibro-tactile stimulus is selectively sensitive to combination of modality and previous experience. Expertise in MFS is reflected in better perceptual abilities of surgeons, suggesting that deliberate practice selectively affected the representation of the audio-tactile stimuli highly relevant to the surgery procedure.

\section{Acknowledgments}

This work was supported by the SKILLS Integrated Project (IST-FP6 \#035005, http://www.skills-ip.eu) funded by the European Commission.

\section{References}

[1] Soto-Faraco S, Deco G. Multisensory contributions to the perception of vibrotactile events. Behav Brain Res. 2009 Jan 23;196(2):145-54. Epub 2008 Sep 30.

[2] M. Ernst, and M. Banks, (2001). "Does vision always dominate haptics?" in Conference on Touch in Virtual Environments, University of Southern California

[3] Bresciani JP, Ernst MO. Signal reliability modulates auditory-tactile integrationfor event counting. Neuroreport 2007;18:1157-61.

[4] Caclin A, Soto-Faraco S, Kingstone A, Spence C. Tactile "capture" of audition. Percept Psychophys 2002;64:616-30.

[5] Murray MM, Molholm S, Michel CM, et al. Grabbing your ear: rapid auditorysomatosensory multisensory interactions in low-level sensory cortices are not constrained by stimulus alignment. Cereb Cortex 2005;15:963-74.

[6] Soto-Faraco S, Spence C, Kingstone A. Congruency effects between auditory and tactile motion: extending the phenomenon of cross-modal dynamic capture. Cogn Affect Behav Neurosci 2004;4:208-17.

[7] Bresciani JP, ErnstMO, Drewing K, Bouyer G,MauryV, KheddarA. Feeling what you hear: auditory signals can modulate tactile tap perception. Exp Brain Res 2005;162:172-80.

[8] Zampini M, Torresan D, Spence C, Murray MM. Auditory-somatosensory multisensory interactions in front and rear space. Neuropsychologia 2007;45:1869-77.

[9] Gillmeister H, Eimer M. Brain Res. Tactile enhancement of auditory detection and perceived loudness. 2007 Jul 30;1160:58-68. Epub 2007 Mar 20. [10] Praamsma M, Carnahan H, Backstein D, Veillette CJ, Gonzalez D, Dubrowski A. Drilling sounds are used by surgeons and intermediate residents, but not novice orthopedic trainees, to guide drilling motions. Can J Surg. 2008 Dec;51(6):442-6.

[11] Korman M, Weiss K, Cohen A, Reiner M, Gopher D. Effects of Practice and Sensory Modality on Stiffness Perception. Presence, 2011 Accepted 